\title{
Probing modified gravitational wave propagation with strongly lensed coalescing binaries
}

\author{
Andreas Finke, ${ }^{1}$ Stefano Foffa, ${ }^{1}$ Francesco Iacovelli, ${ }^{1}$ Michele Maggiore,${ }^{1}$ and Michele Mancarella ${ }^{1}$ \\ ${ }^{1}$ Département de Physique Théorique and Center for Astroparticle Physics, \\ Université de Genève, 24 quai Ansermet, CH-1211 Genève 4, Switzerland
}

\begin{abstract}
It has been recently shown that quadruply lensed gravitational-wave (GW) events due to coalescing binaries can be localized to one or just a few galaxies, even in the absence of an electromagnetic counterpart. We discuss how this can be used to extract information on modified GW propagation, which is a crucial signature of modifications of gravity at cosmological scales. We show that, using quadruply lensed systems, it is possible to constrain the parameter $\Xi_{0}$ that characterizes modified GW propagation, without the need of imposing a prior on $H_{0}$. A LIGO/Virgo/Kagra network at target sensitivity might already get a significant measurement of $\Xi_{0}$, while a third generation GW detector such as the Einstein Telescope could reach a very interesting accuracy.
\end{abstract}

\section{INTRODUCTION}

Gravitational waves (GWs) propagating across cosmological distances are bent by nearby galaxies and galaxy clusters, just as light does. Signatures for lensing of GWs have been searched in the data of the $\mathrm{O} 1$ and $\mathrm{O} 2$ LIGO/Virgo observing runs [1-6] and in the O3a data [7], and no compelling evidence for lensing of GW events has been reported. With current detector sensitivity, the expected fraction of observed strong lensing events is of order $10^{-4}-10^{-3}$, depending on assumptions of the merger rate [7] so, with the $\mathcal{O}(50)$ coalescences detected to date, the observation of a lensed event is unlikely (see [8] for an opposite viewpoint). At design sensitivity, the rate of strongly lensed events for advanced LIGO/Virgo could be around one event per year [9-11] (again, with a significant uncertainty due to the merger-rate density at high redshift). This figure greatly increases for a third generation GW detector such as the Einstein Telescope (ET) $[12,13]$, which could observe $\mathcal{O}(50-150)$ strongly lensed binary black holes (BBHs) per year [14], plus tens to hundreds $\mathrm{BBH}$ events per year and $\mathcal{O}(10-50)$ black hole-neutron star (BH-NS) and neutron star-neutron star (NS-NS) lensed events per year, that would have been below the detection threshold, but rise above the detection threshold because of the lensing magnification [15].

Just as for lensing of electromagnetic signals, which is now a standard tool in astrophysics [16, 17], lensing of GWs could be used as a probe of several effects. In particular, the detection of multiply lensed GW events would provide a new tool for cosmography, allowing a measurement of the Hubble parameter $H_{0}$ and of the dark energy equation of state [18-21]. In this paper we explore another potentially very interesting application of strong lensing of GW events, to the study of modified GW propagation.

The paper is organized as follows. In sect. II we review the phenomenon of modified GW propagation, and the existing observational limits. Strong lensing of GWs is briefly reviewed in sect. III, focusing in particular on the potential for cosmography of quadruply lensed events, recently explored in [22]. In sect. IV we discuss strong lensing in modified gravity, pointing out that quadruply lensed events allow for the simultaneous determination of the standard ('electromagnetic') luminosity distance, and of the 'GW luminosity distance' that characterizes modified GW propagation. In sect. $\mathrm{V}$ we discuss how, in practice, one could use a quadruply lensed event to measure the parameter $\Xi_{0}$ that we use to parametrize modified GW propagation. Sect. VI contains our conclusions.

\section{MODIFIED GW PROPAGATION}

In recent years, modified GW propagation has been understood as a completely generic property of theories that modify General Relativity (GR) on cosmological scales [23-30], and it has been realized that it can be the most promising observable for testing such theories (see, in particular, the discussions in [27, 29, 31]).

The phenomenon is encoded in a modification of the 'friction term' in the equation that governs the propagation of tensor perturbations over a FriedmannRobertson-Walker (FRW) background, that, in modified gravity, can take the form

$$
\tilde{h}_{A}^{\prime \prime}+2 \mathcal{H}[1-\delta(\eta)] \tilde{h}_{A}^{\prime}+k^{2} c^{2} \tilde{h}_{A}=0 .
$$

GR is recovered for $\delta(\eta)=0$ (we use standard notation: $h_{A}$ is the GW amplitude, $A=+, \times$ labels the two polarizations, the prime denotes the derivative with respect to cosmic time $\eta, a(\eta)$ is the FRW scale factor, and $\left.\mathcal{H}=a^{\prime} / a\right)$. We have not included the possibility of modifying the coefficient of the term $k^{2} c^{2}$ term, since this would affect the speed of GWs, and is now excluded, at a level $O\left(10^{-15}\right)$ [32]. Still, even in modified gravity theories that do not change the $k^{2} c^{2}$ term (such as a subset of scalar-tensor theories [33-36], or modifications of GR induced by the generation of non-local terms in the quantum effective action [37-39]), a non-trivial function $\delta(\eta)$ emerges. Indeed, the detailed study in [30] shows that this modification is completely generic, and takes place in all modified gravity models that have been investigated. 
A consequence of eq. (1), with a non-vanishing function $\delta(\eta)$, is that, in the propagation across cosmological distances, the amplitude of GWs is attenuated in a different way, with respect to the $\propto 1 / a$ behavior of GR. Then, GW observations of coalescing binaries no longer measure the luminosity distance $d_{L}(z)$ of the source [that, in this context, we call the 'electromagnetic luminosity distance' and we denote by $\left.d_{L}^{\mathrm{em}}(z)\right]$, but rather a 'GW luminosity distance' $d_{L}^{\mathrm{gw}}(z)$, related to $d_{L}^{\mathrm{em}}(z)$ by $[27,29]$

$$
d_{L}^{\mathrm{gw}}(z)=d_{L}^{\mathrm{em}}(z) \exp \left\{-\int_{0}^{z} \frac{d z^{\prime}}{1+z^{\prime}} \delta\left(z^{\prime}\right)\right\},
$$

where the function $\delta$ that appears in eq. (1) has now been written as a function of redshift.

For comparing with observations it is useful to have a parametrization of this effect in terms of a small number of parameters, rather than a full function $\delta(z)$. In the following we will use the parametrization proposed in [29],

$$
\frac{d_{L}^{\mathrm{gw}}(z)}{d_{L}^{\mathrm{em}}(z)} \equiv \Xi(z)=\Xi_{0}+\frac{1-\Xi_{0}}{(1+z)^{n}},
$$

in terms of two parameters $\left(\Xi_{0}, n\right)$. This parametrization reproduces the fact that, for $z=0, d_{L}^{\mathrm{gw}} / d_{L}^{\mathrm{em}}=1$, as it should, and is such that, in the limit of large redshifts, $d_{L}^{\mathrm{gw}} / d_{L}^{\mathrm{em}}$ goes to a constant value $\Xi_{0}$. This is indeed what happens in most modified gravity models, where the deviations from GR only appear in the recent cosmological epoch; therefore, $\delta(z)$ goes to zero at large redshifts, and the integral in eq. (2) saturates to a constant value. Equation (3) smoothly interpolates between these two limiting behaviors with a power-law that, written in terms of the scale factor $a$, reads, even more simply, $d_{L}^{\mathrm{gw}} / d_{L}^{\mathrm{em}}=\Xi_{0}+a^{n}\left(1-\Xi_{0}\right)$. As shown in ref. [30], eq. (3) fits remarkably well the explicit results from all the best-studied modified gravity models, such as several examples of Horndeski and DHOST theories, or non-local infrared modifications of gravity [with the exception of bigravity, where the result for $d_{L}^{\mathrm{gw}}(z) / d_{L}^{\mathrm{em}}(z)$ displays some oscillations as a function of redshift, rather than the smooth behavior given by eq. (3)]. In the parametrization (3), GR is recovered when $\Xi_{0}=1$ (for all $n$ ).

The next question is what is the size of the deviations from the GR value $\Xi_{0}=1$, that one could expect in viable modified gravity models. In general, to comply with cosmological observations with electromagnetic probes such as cosmic microwave background (CMB), supernovae $(\mathrm{SNe})$, baryon acoustic oscillations $(\mathrm{BAO})$, and structure formation, the deviations from GR and, more specifically, from $\Lambda \mathrm{CDM}$, are bounded at the level of a few percent, for both the background evolution and the scalar perturbations. One might then expect that also the deviations for tensor perturbations (i.e., for GWs propagating in a FRW background) will be of the same order, leading at most to a deviation of $\Xi_{0}$ from 1 by a few percent. However, the study of explicit models shows that this is not necessarily the case, and the deviations in the tensor sector can be much larger. A remarkable example is given by the so-called RT non-local gravity model [37] (see [39] for an updated review). This model is very close to $\Lambda \mathrm{CDM}$ in the background and in the scalar perturbations, and indeed fits cosmological observations at a level statistically equivalent to $\Lambda$ CDM. However, it predicts that $\Xi_{0}$ can be as large as $1.80[39,40]$, corresponding to a $80 \%$ deviation from the GR value $\Xi_{0}=1$. Independently of the specific virtues of this model (which has an appealing field-theoretical motivation, with nonlocal terms generated by infrared quantum effects in the quantum effective action, generates dynamically a dark energy, and fits current observations at the same level as $\Lambda \mathrm{CDM})$, this can be taken just as an example of the fact that phenomenologically viable models can predict large deviations from $\Lambda \mathrm{CDM}$ in the sector of tensor perturbations, which is the new cosmological window that GW experiments are beginning to open.

An observational limit on modified GW propagation was obtained in [29] from GW170817, comparing the GW luminosity distance, obtained from the GW observation, to the electromagnetic luminosity distance of the galaxy hosting the counterpart, obtained from surface brightness fluctuations. Given the small redshift of GW170817, this is really a measurement of $\delta(z=0)$, independently of any parametrization, and gives $\delta(0)=-7.8_{-18.4}^{+9.7}$, which is of course consistent with the GR prediction $\delta(z)=$ 0 . Setting a value $n \simeq 2$ (typical for instance of the predictions of nonlocal gravity) and using $\delta(0)=n(1-$ $\Xi_{0}$ ), this can be translated into a bound $\Xi_{0} \lesssim 14$ (see also $[26,41]$ for other analysis using GW170817, resulting in somewhat broader but consistent limits).

Recently, a more stringent limit has been obtained in [31], using BBH coalescences from the O1, O2 and O3a LIGO/Virgo run and correlating them with the GLADE galaxy catalog [42]. The result is $\Xi_{0}=2.1_{-1.2}^{+3.2}$ ( $68 \%$ c.l.). This shows that interesting limits on $\Xi_{0}$ can already be obtained with current GW observations. An even more stringent result is obtained under the tentative identification of the flare ZTF19abanrhr as the electromagnetic counterpart of the BBH coalescence GW190521, in which case the result obtained in [31] is $\Xi_{0}=1.8_{-0.6}^{+0.9}$ (see also [43] for a related result, and [44] for forecasts on the limits that could be obtained with $\mathcal{O}(100)$ BNS). Modified GW propagation has also been recently constrained by combining GW data with the mass distribution of compact binaries [45], following the strategy proposed in [46].

For third-generation detectors such as the Einstein Telescope (ET) [12, 13] and Cosmic Explorer [47], or for the LISA space interferometer [48], the perspectives are quite exciting. In [29, 49], using state-of-the-art mock catalogs for the GW events and for the detection of an associated GRB, it is estimated that ET could measure $\Xi_{0}$ to about $1 \%$ (depending on the network of GRB satellites available at the time when ET will operate). Forecasts for LISA have been presented in [30], using the coalescence of supermassive black holes (SMBH), and it has been found that $\Xi_{0}$ could be measured to $(1-4) \%$ accu- 
racy. Forecasts for the correlation between LISA SMBH events and the prediction of the Horndeski theory for large scale structure have been presented in [50].

In the following we will discuss another possible way of searching for modified GW propagation, using strongly lensed GW events.

\section{STRONG LENSING OF GW EVENTS}

In GW astronomy the angular resolutions of the detectors are orders of magnitude above the arcsec resolution needed to spatially separate typical lensed images. However, the signals from coalescing binaries have a short duration. Therefore, strong lensing will rather manifest itself through repeated GW detections, associated to signals reaching the observer through different paths from the same source. These events will have a relative time delay, of orders of minutes to months for lensing by galaxies and up to years for lensing by galaxy clusters, and a different amplitude, which reflects the fact that signals traveling through different paths undergo different amount of magnification or demagnification. The other parameters entering the waveform, such as the detector frame masses, spins, sky locations, etc., are the same (except, possibly, for a frequency-independent phase shift), and a Bayesian analysis can be performed to compare the hypothesis that two (or more) GW events belong to the same source with the hypothesis that they are unrelated [1-6, 51-53].

The possibility of using strongly lensed GW events with an electromagnetic counterpart to perform cosmography has been discussed in $[19,20]$, but BBH coalescences are not expected, in general, to have electromagnetic counterparts. However, as shown in [22], quadruply lensed events (which are estimated to be about $30 \%$ of the total lensed events at LIGO/Virgo, and $6 \%$ for ET [10]) are especially interesting, because they can be used as standard sirens even in the absence of an associated electromagnetic flare, by comparing the lensing pattern obtained from GW observations with that observed electromagnetically. The reason is that quadruply lensed systems have three independent time delays and three independent magnification ratios, and this provides sufficient information to reconstruct the source-lens system from the GW observations. The corresponding lensing pattern must then show up in the electromagnetic observation of the source-lens system. Assuming that the GW event originates from a galaxy that emits electromagnetic radiation, and that (possibly with dedicated follow-up) we know from electromagnetic observations all the strong lensing systems in the localization area of the GW event, taken to be given by the localization resolution of a LIGO/Virgo/KAGRA network at target sensitivity, ref. [22] shows that it is possible to localize the GW source to a single, or at most a few, galaxies. Spectroscopic follow-up could then provide an accurate determination of the redshifts $z_{l}$ of the lens and $z_{s}$ of the source. Once identified the GW host galaxy, a detailed lens model can be used to de-lens the system and infer the absolute magnifications $\mu_{i}$ associated with the distinct images.

According to gravitational lensing theory (see e.g. [16, $17,54])$, the time delay between two 'images' $i$ and $j$ is given by

$$
c \Delta t_{i, j}=\left(1+z_{l}\right) D_{\Delta t}\left(z_{l}, z_{s}\right) \Delta \phi_{i, j},
$$

where $\Delta \phi_{i, j}$ is the difference of the Fermat potentials between the $i$ and $j$ images, which is obtained from the lens reconstruction, and

$$
D_{\Delta t}\left(z_{l}, z_{s}\right)=\frac{d_{A}\left(z_{l}\right) d_{A}\left(z_{s}\right)}{d_{A}\left(z_{l}, z_{s}\right)}
$$

where $d_{A}\left(z_{l}\right), d_{A}\left(z_{s}\right)$ and $d_{A}\left(z_{l}, z_{s}\right)$ are the angular diameter distances from the lens to the observer, from the source to the observer, and from the lens to the source, respectively. Furthermore, the observed luminosity distance extracted from the $i$-th lensed image, $D_{i}$, is related to the actual, unlensed, luminosity distance $d_{L}$ of the source by

$$
D_{i}=d_{L} / \sqrt{\mu_{i}} .
$$

Since the ratio of time delays, and the ratio of magnifications $\mu_{i} / \mu_{j}$, are sufficient to reconstruct the source position, the absolute scale of the time delays and of the absolute magnifications can be used to perform inference on the cosmology. This strategy has been used in [22] to forecast the accuracy that could be obtained on $H_{0}$, in the context of $\Lambda \mathrm{CDM}$, with a LIGO/Virgo/KAGRA network.

\section{STRONG LENSING IN MODIFIED GRAVITY}

In this paper we examine the information that can be obtained on $\Xi_{0}$, in the context of modified gravity, from a quadruply lensed GW event. The crucial observation is that, in modified gravity, eq. (6) becomes

$$
D_{i}=d_{L}^{\mathrm{gw}} / \sqrt{\mu_{i}},
$$

since $d_{L}^{g \mathrm{~g}}$ is the quantity that encodes the attenuation of the GW amplitude in the propagation across cosmological distances. In contrast, the angular diameter distances that appear in eq. (5) are the same for GWs and for electromagnetic waves, and have nothing to do with the damping of the GW amplitude during their propagation. These angular diameter distances are therefore related to the standard ('electromagnetic') luminosity distance by the usual relation $d_{A}(z)=d_{L}^{\mathrm{em}}(z) /(1+z)^{2}$. In particular, recalling that (in a spatially flat universe)

$$
d_{A}\left(z_{l}, z_{s}\right)=d_{A}\left(z_{s}\right)-\frac{1+z_{l}}{1+z_{s}} d_{A}\left(z_{l}\right),
$$


we can rewrite $D_{\Delta t}\left(z_{l}, z_{s}\right)$ in terms of the electromagnetic luminosity distances of the source and of the lens, as

$D_{\Delta t}\left(z_{l}, z_{s}\right)=\frac{d_{L}^{\mathrm{em}}\left(z_{l}\right) d_{L}^{\mathrm{em}}\left(z_{s}\right)}{\left(1+z_{l}\right)^{2} d_{L}^{\mathrm{em}}\left(z_{s}\right)-\left(1+z_{l}\right)\left(1+z_{s}\right) d_{L}^{\mathrm{em}}\left(z_{l}\right)}$,

(see also [55], where a study of the viscosity term was carried out for GW events with counterpart). We write

$$
d_{L}^{\mathrm{em}}(z)=\frac{1+z}{H_{0}} \int_{0}^{z} \frac{d \tilde{z}}{E(\tilde{z})}
$$

where

$$
E(z)=\left[\Omega_{R}(1+z)^{4}+\Omega_{M}(1+z)^{3}+\rho_{\mathrm{DE}}(z) / \rho_{0}\right]^{1 / 2},
$$

$\rho_{0}=3 H_{0}^{2} /(8 \pi G), \Omega_{R}$ and $\Omega_{M}$ are the radiation and matter density fractions, respectively, while $\rho_{\mathrm{DE}}(z)$ is the dark energy (DE) density. In $\Lambda \mathrm{CDM} \rho_{\mathrm{DE}}(z) / \rho_{0}=\Omega_{\Lambda}$ is a constant, while in a generic modified gravity theory

$$
\rho_{\mathrm{DE}}(z) / \rho_{0}=\Omega_{\mathrm{DE}} e^{3 \int_{0}^{z} \frac{d \tilde{z}}{1+\tilde{z}}\left[1+w_{\mathrm{DE}}(\tilde{z})\right]},
$$

where $\Omega_{\mathrm{DE}}=\rho_{\mathrm{DE}}(0) / \rho_{0}$, and $w_{\mathrm{DE}}(z)$ the $\mathrm{DE}$ equation of state. Using eq. (10), eq. (9) can be rewritten as

$$
D_{\Delta t}\left(z_{l}, z_{s}\right)=\frac{R\left(z_{l}, z_{s}\right)}{\left(1+z_{l}\right)\left(1+z_{s}\right)} d_{L}^{\mathrm{em}}\left(z_{s}\right)
$$

where

$$
R\left(z_{l}, z_{s}\right)=\frac{\int_{0}^{z_{l}} d z^{\prime} / E\left(z^{\prime}\right)}{\int_{z_{l}}^{z_{s}} d z^{\prime} / E\left(z^{\prime}\right)}
$$

Observe that $H_{0}$ canceled in the ratio $R\left(z_{l}, z_{s}\right)$ which therefore depends on the cosmology only through $\Omega_{M}$ and, in modified gravity, on the DE equation of state, that enters in $E(z)$ through $\rho_{\mathrm{DE}}(z)$.

To sum up, from a quadruply lensed GW signal, we can get a reconstruction of the redshift of the source and of the lens, four measurements of $d_{L}^{\text {gw }}$ from the amplitude of the four images, and a measurement of the combination (9), that (given $z_{l}, z_{s}$ and the cosmology) only involves the electromagnetic luminosity distance of the source. The DE sector of the theory enters through the function $w_{\mathrm{DE}}(z)$ that affects $d_{L}^{\mathrm{em}}(z)$ according to eqs. (10)-(12), and through the function $\Xi(z)$ that, given $d_{L}^{\mathrm{em}}(z)$, determines $d_{L}^{\mathrm{gw}}(z)$ according to eq. (3). Therefore, given the (four) observed values of $d_{L}^{\mathrm{gw}}$ and the observed value of $D_{\Delta t}\left(z_{l}, z_{s}\right)$, we can in principle perform an inference on $w_{\mathrm{DE}}(z)$, using for instance the $\left(w_{0}, w_{a}\right)$ parametrization of the redshift dependence $[56,57], w_{\mathrm{DE}}(z)=w_{0}+[z /(1+z)] w_{a}$, and on $\Xi(z)$, for which we will use the $\left(\Xi_{0}, n\right)$ parametrization $(3)$.

In the following, to simplify the analysis, we will fix $w_{\mathrm{DE}}=-1$ (i.e. $w_{0}=-1, w_{a}=0$ ), as in $\Lambda$ CDM, and we will only consider $\Xi_{0}$ as a free parameter. ${ }^{1}$ This is motivated by the fact that we know, from cosmological observations with electromagnetic probes such as cosmic microwave background (CMB), supernovae (SNe), baryon

\footnotetext{
1 The parameter $n$ in eq. (3) describes the precise power-law be-
}

acoustic oscillations (BAO), structure formation, etc., that $w_{\mathrm{DE}}$ cannot differ from -1 by more than about $5 \%$. In contrast, the current limit on $\Xi_{0}$, that can come only from GW observations, are much broader. As mentioned in sect. II, the correlation of $\mathrm{O} 1+\mathrm{O} 2+\mathrm{O} 3 \mathrm{a}$ dark sirens with the GLADE galaxy catalog gives $\Xi_{0}=2.1_{-1.2}^{+3.2}$, which still allows (at $1 \sigma$ ) values of $\Xi_{0}$ as large as $\Xi_{0} \simeq 5$, i.e. deviations from the GR value at the level of $500 \%$. The most stringent values obtained in [31, 43] are based on the assumption that the flare ZTF19abanrhr is the counterpart of the BBH coalescence GW190521, an identification which is not secure, and in any case still allow deviations at the $300 \%$ level from GR. As we have mentioned above, a phenomenologically viable model such as the RT nonlocal model predicts a value of $\Xi_{0}$ that can be as large as 1.80, i.e. a $80 \%$ deviation from GR.

Furthermore, as discussed in [29], the accuracy that can be reached on $\Xi_{0}$ from standard sirens is in general better than the corresponding accuracy on $w_{0}$. This is due to to the fact that the effect of $w_{0}$ on the electromagnetic luminosity distance is masked by partial degeneracies with $H_{0}$ and $\Omega_{M}$ : moving $w_{0}$ away from -1 , the value of $H_{0}$ and $\Omega_{M}$ (which are determined by fitting the modified model to cosmological data such as CMB, BAO and $\mathrm{SNe}$ ) change, with respect to their values in $\Lambda \mathrm{CDM}$, and precisely in the direction to partially compensate the effect of the change of $w_{0}$ in the luminosity distance.

For these reasons, it makes sense to consider a phenomenological scenario where the deviation of $\Xi_{0}$ from 1 are large, while $w_{\mathrm{DE}}$ is very close to -1 (and the RT nonlocal model provides an explicit realization of this scenario). Note that, in contrast, when studying cosmography with GWs, it is not really meaningful to consider $w_{0}$ as a free parameter, while neglecting modified $\mathrm{GW}$ propagation. Whenever a dynamical DE model predicts $w_{0} \neq-1$, it also predicts $\Xi_{0} \neq 1$, and the latter effect in general dominates, possibly by even one or two orders of magnitude. ${ }^{2}$

havior between the asymptotic regimes at small and large $z$ and its precise value is less important. When producing the plots in sect. $\mathrm{V}$, we will fix it for definiteness to the value $n=1.91$, which is the value predicted by the RT model in the same limit in which $\Xi_{0}=1.80$.

2 In [58] it has been suggested that, in models that require screening (which does not include the RT nonlocal gravity model) the screening mechanism could also eliminate any detectable modification of the luminosity distance. This, however, applies only in theories where the Newton constant that describes the coupling of matter is the same as the effective Newton constant that appears in the quadratic self-interaction term of gravitational waves, which is not the case in generic modified gravity theories [50]. 


\section{EXTRACTING $\Xi_{0}$ FROM QUADRUPLY LENSED EVENTS}

We now discuss how, in practice, modified GW propagation could be tested from a quadruply lensed system. The first obvious possibility is to use the redshift $z_{s}$ of the source, obtained from the reconstruction of the source-lens system and possibly a dedicated spectroscopic follow-up (in which case the error on $z_{s}$ would be negligible with respect to the other uncertainties), assume a value of $H_{0}$ (and of $\Omega_{M}$ ), and infer from this the electromagnetic luminosity distance to the source, $d_{L}^{\mathrm{em}}\left(z_{s}\right)$. We can then compare it with the GW measurement of $d_{L}^{\mathrm{gw}}\left(z_{s}\right)$, obtained combining the four measurements of the amplitudes $D_{i}$ in eq. (7), with the $\mu_{i}$ given by the reconstruction of the lens system. The posteriors for $d_{L}^{\mathrm{gw}}\left(z_{s}\right)$ and $d_{L}^{\mathrm{em}}\left(z_{s}\right)$ could then be transformed into a posterior $P\left[\left(\Xi\left(z_{s}\right)\right]\right.$ for $\Xi\left(z_{s}\right)$ and therefore (assuming a given $n$ ) into a posterior for $\Xi_{0}$, obtained from

$$
\begin{aligned}
P\left(\Xi_{0}\right) & =P\left[\left(\Xi\left(z_{s}\right)\right] \frac{d \Xi\left(z_{s}\right)}{d \Xi_{0}}\right. \\
& =P\left[\left(\Xi\left(z_{s}\right)\right]\left[1-\frac{1}{\left(1+z_{s}\right)^{n}}\right] .\right.
\end{aligned}
$$

More generally, we can express the result in terms of a posterior in the $\left(\Xi_{0}, n\right)$ plane.

The disadvantage of this procedure is that we need to assume a value for $H_{0}$. Even neglecting for a moment the tension between early-Universe $[59,60]$ and late Universe [61-63] measurements of $H_{0}$, and focusing on early Universe measurement only, still the value of $H_{0}$ (and of $\Omega_{M}$ ), determined by fitting the cosmological parameters to $\mathrm{CMB}, \mathrm{BAO}$ and $\mathrm{SNa}$ data, depends in principle on the modified gravity theory considered. This might not be such a problem, at least as long as we look for large deviations of $\Xi_{0}$ from the GR value, since typical modified gravity models (or, at least, those based on late-time DE) that fit current cosmological data predict a value of $H_{0}$ quite close to that of $\Lambda \mathrm{CDM}$. For instance, in the RT nonlocal model, the mean values of $H_{0}$ and $\Omega_{M}$ obtained from a fit to $\mathrm{CMB}, \mathrm{BAO}$ and $\mathrm{SNa}$ data differ from that obtained in $\Lambda \mathrm{CDM}$ by less than $0.1 \%$. Nevertheless, in view of the discrepancy between early- and late-Universe measurements of $H_{0}$, it would be even more interesting to have a determination of $\Xi_{0}$ that is independent on assumptions on $H_{0}$.

This is indeed possible, thanks to the extra information carried by $D_{\Delta t}\left(z_{l}, z_{s}\right)$ : according to eq. (13), after lens reconstruction, a measurement of $D_{\Delta t}\left(z_{l}, z_{s}\right)$ determines directly $d_{L}^{\mathrm{em}}\left(z_{s}\right)$ (assuming that $w_{\mathrm{DE}}$ is sufficiently close to $w_{\mathrm{DE}}=-1$, so that we can simply set $w_{\mathrm{DE}}=-1$, at least as long as we search for values of $\Xi_{0}$ significantly different from the GR value $\Xi_{0}=1$; otherwise, we will have to perform a joint inference on $\Xi_{0}$ and $w_{0}$ ).

Of course, the accuracy of the measurement that one can obtain for $d_{L}^{\mathrm{gw}}$ and for $D_{\Delta t}\left(z_{l}, z_{s}\right)$ strongly depends on the network of detectors under consideration, and will also in general change significantly from event to event.
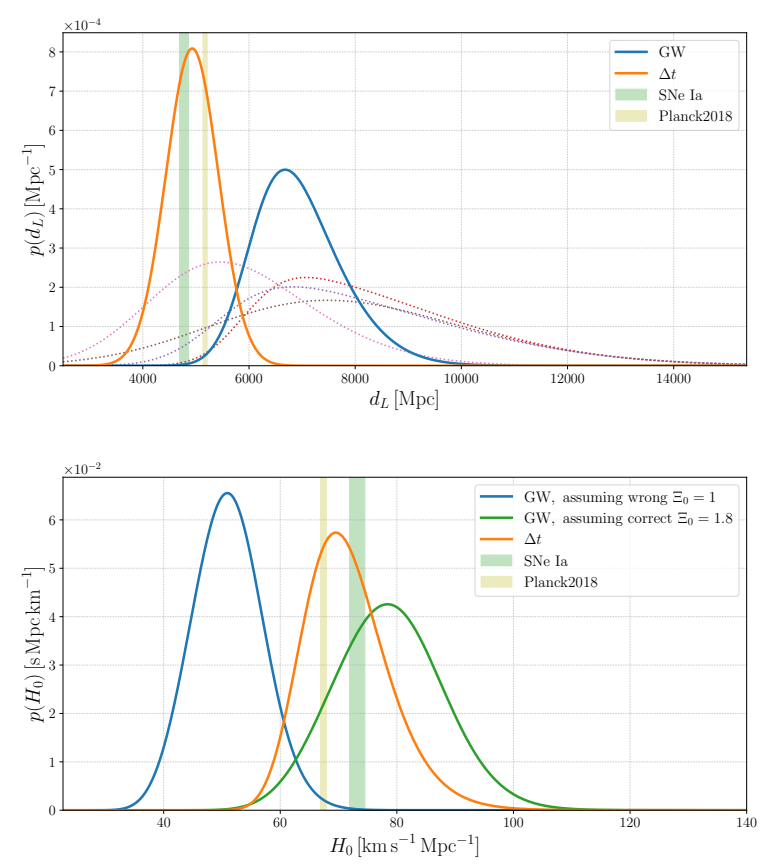

FIG. 1. Upper plot: posterior for $d_{L}$ as the result of combining a quadruple lensed GW (solid blue, each individual image represented by the dotted curves), and the measurement of time delays (orange). Lower plot: the same information translated into a posterior for $H_{0}$, assuming $\Lambda$ CDM (blue) or a modified gravity model (green).

As an illustration of the procedure, we first consider an accuracy on these measurements of the order of that obtained from the detailed analysis in [22] (see in particular their Fig. 5), which assumes a LIGO/Virgo/Kagra network at target sensitivity, simulates a lens distribution that follow a given galaxy-galaxy lens distribution [64], randomly simulates GW events that are quadruply lensed and pass a network detector threshold $\rho_{\text {thre }}>10$, and performs the lens reconstruction.

The upper panel of Fig. 1 shows the kind of posteriors that one could expect. To produce this plot we have assumed that the fiducial values of the cosmological parameters are $H_{0}=70 \mathrm{~km} \mathrm{~s}^{-1} \mathrm{Mpc}^{-1}, \Omega_{M}=0.31$ and $\Xi_{0}=1.8$. We are therefore assuming that the correct theory of gravity is a modified gravity theory with a large deviation from $\Xi_{0}=1$, inspired by the value found in the RT nonlocal model. We also assume that the 'true' redshift of the source is $z_{s}=0.8$. We have then computed the 'true' values of $d_{L}^{\mathrm{em}}\left(z_{s}\right)$ and $d_{L}^{\mathrm{gw}}\left(z_{s}\right)$ with our fiducial values of the cosmological parameters. The 'observed' mean value $\mu_{\mathrm{em}}$ of the luminosity distance $d_{L}^{\mathrm{em}}$, obtained from $D_{\Delta t}\left(z_{l}, z_{s}\right)$, has been drawn from a gaussian with mean $d_{L}^{\mathrm{em}}\left(z_{s}\right)$ and standard deviation $\sigma=0.1 d_{L}^{\mathrm{em}}\left(z_{s}\right)$, to simulate a scattering of the observed value with respect to the true value. For the posterior of $d_{L}^{\mathrm{em}}$, shown as the orange line in the upper panel of Fig. 1, we have assumed again a gaussian shape, as suggested 
by the result in [22], centered on $\mu_{\mathrm{em}}$ and with the same width $\sigma=0.1 d_{L}^{\mathrm{em}}\left(z_{s}\right)$, i.e. a measurement of the distance at the $10 \%$ level. The green and light green bands show the result that would be obtained for $d_{L}^{\mathrm{em}}\left(z_{s}\right)$ using the value $H_{0}=67.4 \pm 0.5 \mathrm{~km} \mathrm{~s}^{-1} \mathrm{Mpc}^{-1}$ from Planck 2018 [59] and the value $H_{0}=73.2 \pm 1.3 \mathrm{~km} \mathrm{~s}^{-1} \mathrm{Mpc}^{-1}$ from SNe Ia [63], respectively. Our fiducial value is $H_{0}=70 \mathrm{~km} \mathrm{~s}^{-1} \mathrm{Mpc}^{-1}$, somewhat in the middle of them, and the random scattering that we have added happens to move the orange posterior closer to the SNe Ia value.

For $d_{L}^{\mathrm{gw}}$, the distribution of the individual measurements from each of the four images (dashed lines in the upper panel of Fig. 1) are now taken to be skewed gaussians, again as suggested by the results in Fig. 5 of [22]. For the mean values $\left(\mu_{\mathrm{gw}}\right)_{i}$, we proceed as for $d_{L}^{\mathrm{em}}$. The standard deviation and skewness of the dashed lines are each drawn from a uniform distributions (between $40 \%$ and $60 \%$ relative error for the standard deviation, and between 0.5 and 8 for the skewness). The blue solid line is then the combination of these four distributions. Even if the separate distributions are skewed gaussians, the combined posterior for the four events is already fairly gaussian. The mean value for $\mu_{\mathrm{gw}}$ of the combined distribution is of course sensibly different from $\mu_{\mathrm{em}}$, since we have taken $\Xi_{0}=1.8$, and the quantity that is measured by the GW amplitude is a GW luminosity distance, not an electromagnetic luminosity distance.

Of course, when analyzing the data from a quadruply lensed system, one would first start by assuming the validity of GR, and therefore one would treat both the measurement from $D_{\Delta t}\left(z_{l}, z_{s}\right)$ and the measurement from the four separate amplitudes as measurements of the same quantity $d_{L}\left(z_{s}\right)$. The signal of a deviation from GR would then be given by the observation that the combined posterior distribution from the four separate amplitudes, blue line in the upper panel of Fig. 1, is not consistent, to a given statistical significance, with the posterior obtained from $D_{\Delta t}\left(z_{l}, z_{s}\right)$, orange line in the upper panel of Fig. 1. In the lower panel of Fig. 1 we show the corresponding posteriors of $H_{0}$ that would be obtained from the posteriors of $d_{L}\left(z_{s}\right)$, using the fact that $z_{s}$ has been determined by the lensing reconstruction (assuming negligible error on $z_{s}$, thanks to a spectroscopic follow up), and assuming the validity of GR and (spatially flat) $\Lambda$ CDM, i.e. using eq. (10) with $\rho_{\mathrm{DE}}(z) / \rho_{0}=\Omega_{\Lambda}$ and our fiducial value of $\Omega_{M}$. In this case, the signal of deviation from GR and $\Lambda$ CDM would be given by the discrepancy between the blue curve, obtained from the GW amplitudes assuming $\Xi_{0}=1$, and the orange curve, obtained from $D_{\Delta t}\left(z_{l}, z_{s}\right)$. The green curve is the result from the measurement of the four GW amplitudes, once one uses our fiducial value $\Xi_{0}=1.8$, and we see that the consistency with the posterior from $D_{\Delta t}\left(z_{l}, z_{s}\right)$ is re-established (within a small difference that reflects the scatter in $\mu_{\mathrm{gw}}$ and $\mu_{\mathrm{em}}$ that we have introduced to simulate an actual observational situation).

After being alerted on the possibility of deviations from GR by a certain level of inconsistency between these pos-

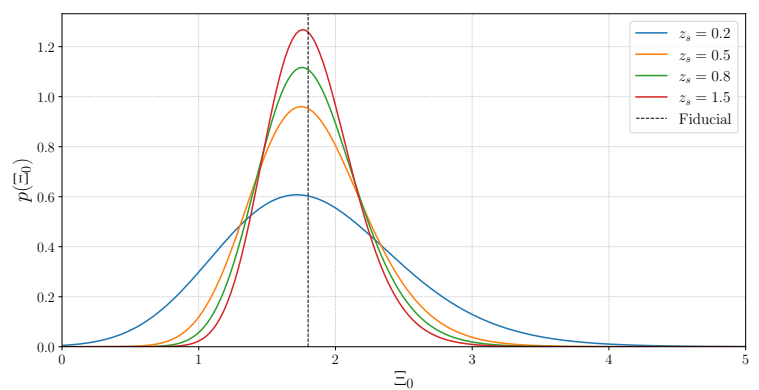

FIG. 2. Examples of posteriors for $\Xi_{0}$ for different values of the source redshift $z_{s}$, corresponding to different possible measurements for a fiducial model with $\Xi_{0}=1.8$.

teriors, one would then naturally open up the parameter space to include $\Xi_{0}$, interpreting the combined measurement from the four amplitudes as a determination of $d_{L}^{\mathrm{gw}}\left(z_{s}\right)$, and the measurement from $D_{\Delta t}\left(z_{l}, z_{s}\right)$ as a determination of $d_{L}^{\mathrm{em}}\left(z_{s}\right)$. From this, one would then get a posterior for $\Xi\left(z_{s}\right)=d_{L}^{\mathrm{gw}}\left(z_{s}\right) / d_{L}^{\mathrm{em}}\left(z_{s}\right)$, and then for $\Xi_{0}$, as in eq. (15). The corresponding result is shown in Fig. 2, where we show the posterior obtained from the simulated source at $z_{s}=0.8$ already used in Fig. 1 (except that here we do not add a random displacement, so that the median of the distribution is now centered exactly on our fiducial value $\Xi_{0}=1.8$ ), as well as the posteriors obtained with the same procedure for sources at different values of $z_{s}$. For all four redshifts in the plot, we have assumed the same fractional error on the measurements of $D_{\Delta t}\left(z_{l}, z_{s}\right)$ and on the GW luminosity distance, as in the case $z_{s}=0.8$. We see that, at fixed relative error in the measurement, the posterior for $\Xi_{0}$ becomes more and more narrow increasing $z_{s}$, since the ratio $d_{L}^{\mathrm{gw}}(z) / d_{L}^{\mathrm{em}}(z)$ increases with $z$, at least before saturating at the asymptotic value $\Xi_{0}$. Observe also that, since we did not add any random displacement, the medians of the posteriors in Fig. 2 coincide with the fiducial value of $\Xi_{0}$. In reality, instrument noise and uncertainties in the reconstruction will displace both the posteriors of $d_{L}^{\mathrm{em}}\left(z_{s}\right)$ and of $d_{L}^{\mathrm{gw}}\left(z_{s}\right)$, and half of the events (when the peaks are moved apart from each other by chance) will therefore provide more tension with the GR value $\Xi_{0}=1$ than what we illustrate, while the other half will provide less tension.

Fig. 2 has been made just as an illustration of the procedure for sources at a few given redshifts and given observational errors on the the electromagnetic and gravitational luminosity distances. To have a more general understanding, we repeat the analysis on a grid of values of source redshifts $z_{s}$ and different values for the relative errors on the measurement of the GW luminosity distance from the combined four GW amplitudes, $\left(\Delta d_{L} / d_{L}\right)_{\mathrm{GW}}$, and of the error on the electromagnetic luminosity distance from $D_{\Delta t}\left(z_{l}, z_{s}\right),\left(\Delta d_{L} / d_{L}\right)_{\Delta t}$. Actually, in a first approximation, the result depends mainly on the sum of 


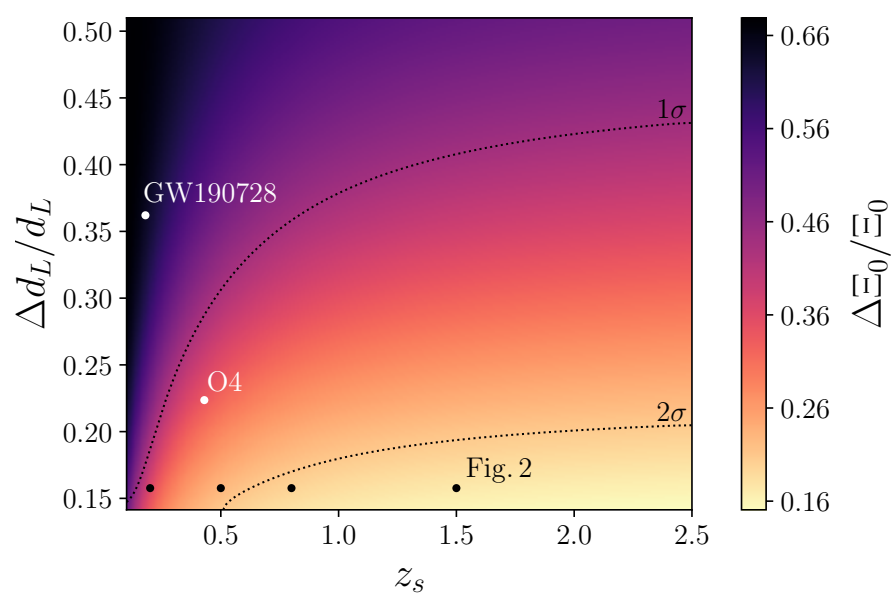

FIG. 3. Colormap plot of the precision attainable on $\Xi_{0}$ as a function of the redshift and of the combined relative error of the measurements coming from the GW amplitude and arrival times, assuming $\Xi_{0}=1.8$ as fiducial value. See the text for the meaning of the different dots and lines.

the relative errors in quadrature,

$$
\frac{\Delta d_{L}}{d_{L}} \equiv\left[\left(\frac{\Delta d_{L}}{d_{L}}\right)_{\mathrm{GW}}^{2}+\left(\frac{\Delta d_{L}}{d_{L}}\right)_{\Delta t}^{2}\right]^{1 / 2} .
$$

In the following, we fix $\left(\Delta d_{L} / d_{L}\right)_{\Delta t}=10 \%$ and we vary $\left(\Delta d_{L} / d_{L}\right)_{\mathrm{GW}}$, but the result applies to any other combinations of $\left(\Delta d_{L} / d_{L}\right)_{\Delta t}$ and $\left(\Delta d_{L} / d_{L}\right)_{\mathrm{GW}}$ that gives the same total $\Delta d_{L} / d_{L}$.

In Fig. 3 we show the result for the relative error $\Delta \Xi_{0} / \Xi_{0}$ in the plane $\left(z_{s}, \Delta d_{L} / d_{L}\right)$, where, for each point, $\Delta \Xi_{0}$ is the $68 \%$ confidence interval of the distribution of $\Xi_{0}$, and $\Xi_{0}$ is the median of the distribution, and we use a range of redshifts appropriate to $2 \mathrm{G}$ detectors (note that lensing magnification can somewhat extend their range). In the figure, the black dots correspond to the examples shown in Fig. 2. The white dot labeled GW190728_064510 corresponds to the best localized event among the most likely lensed pair candidates quoted in [7]; in this case only the error $\left(\Delta d_{L} / d_{L}\right)_{\mathrm{GW}}$ has been included. The white dot labeled O4 corresponds to an example of an event that could be expected in the $\mathrm{O} 4 \mathrm{run}$, assuming $\left(\Delta d_{L} / d_{L}\right)_{\mathrm{GW}}=20 \%$ and $\left(\Delta d_{L} / d_{L}\right)_{\Delta t}=10 \%$, and taking $z_{s}=0.4$, which is the average detection redshift for LIGO in $\mathrm{O} 4$ for an equal mass $\mathrm{BBH}$ of $30 \mathrm{M}_{\odot}$ (see Tab. 2 of [65]). The dotted lines set the limit for the $1 \sigma$ and $2 \sigma$ exclusion of the GR value $\Xi_{0}=1$, for our fiducial value $\Xi_{0}=1.8$, and when not adding random displacements for the measured distances as mentioned above. Given that the value $\Xi_{0}=1.8$ is already the highest currently obtained from a viable cosmological model, for a single quadruple lensed event the results for $\Xi_{0}$ start to be interesting only in the region of the plane below the $1 \sigma$ line, and only below the $2 \sigma$ line one could start to have some hint for deviations from GR. As mentioned, for every second event, on average,

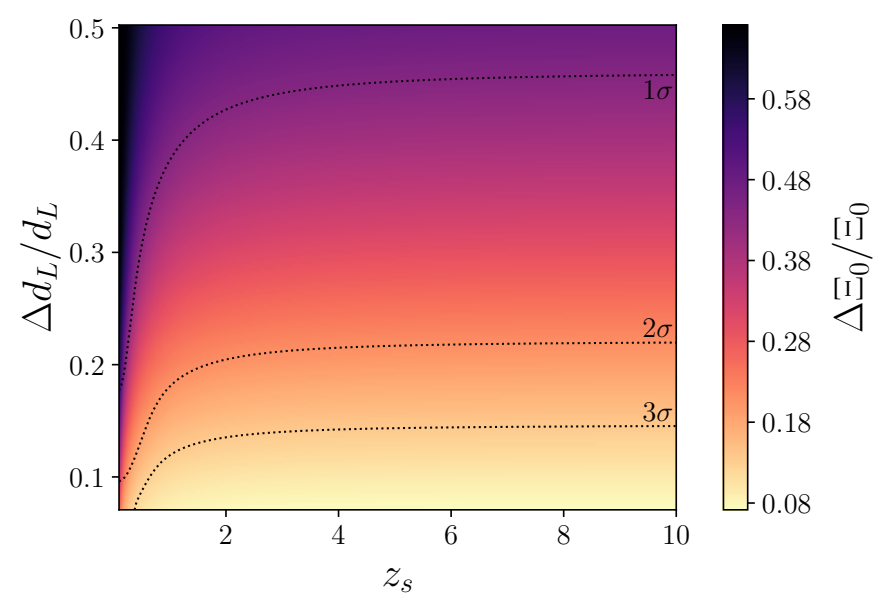

FIG. 4. As in Fig. 3, for a range of redshifts more appropriate to Einstein Telescope.

the median measurement of $\Xi_{0}$ will be larger than 1.8 due to noise. This has the effect of shifting the $1 \sigma$ and $2 \sigma$ lines upwards, which offers the chance of detecting modified gravitational wave propagation with higher significance than shown here. Observe also that, of course, with $N$ lensed events which give a comparable value of $\Delta \Xi_{0} / \Xi_{0}$, the combined error will be further reduced by a factor of order $1 / \sqrt{N}$.

Fig. 4 shows again $\Delta \Xi_{0} / \Xi_{0}$, for a range of redshifts and of $\Delta d_{L} / d_{L}$ more appropriate to a $3 \mathrm{G}$ detectors such as the Einstein Telescope (which can actually even reach much higher redshifts, for which, however, a dedicated study of lensing reconstruction should be performed). A first order of magnitude estimate for the value of $\Delta \Xi_{0} / \Xi_{0}$ that ET could reach from quadruply lensed events can be obtained observing that at ET, and even more with a network involving another third-generation (3G) detector such as Cosmic Explorer, the error $\Delta d_{L} / d_{L}$ will likely be dominated by the lens reconstruction. If, as a first estimate, we assume $\Delta d_{L} / d_{L} \sim 25 \%$, we see from Fig. 3 that, at the large redshifts explored by $3 \mathrm{G}$ detectors, this corresponds to $\Delta \Xi_{0} / \Xi_{0} \sim 0.3$. Such a $30 \%$ accuracy would already be sufficient to test the prediction $\Xi_{0} \simeq 1.8$ from nonlocal gravity with an interesting accuracy, with a single quadruply lensed event. Furthermore, assuming, at ET, 100 strong lensing events per year [14], of which $6 \%$ will be quadruply lensed [10], in four years ET could collect $\mathcal{O}(25)$ quadruply lensed events, so the combined error would be of order $\Delta \Xi_{0} / \Xi_{0} \sim 0.3 / \sqrt{25}=6 \%$.

Note that the case discussed at the beginning of this section, where $d_{L}^{\mathrm{em}}$ is determined from the redshift $z_{s}$ assuming a value of $H_{0}$ and $\Omega_{M}$, can also be included as a special case of Figs. 3 or 4: simply, in this case the error $\left(\Delta d_{L} / d_{L}\right)_{\Delta t}$ in eq. (16) is replaced by the error on $d_{L}^{\mathrm{em}}\left(z_{s}\right)$ obtained from the redshift and the cosmological parameters. If the redshift is accurately known from a spectroscopic follow-up, the error is dominated by the error on the assumed value of $H_{0}$ and $\Omega_{M}$, which, using the 

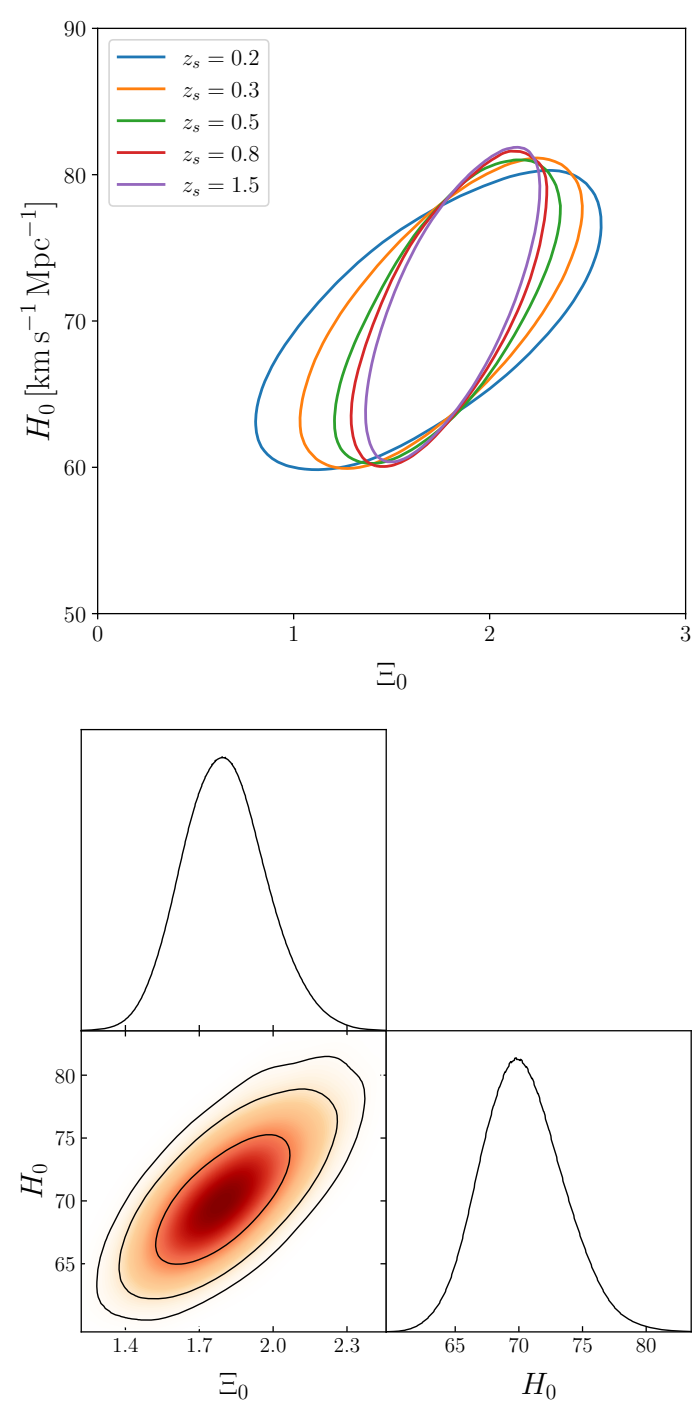

FIG. 5. Upper plot: $68 \%$ contours of events at different redshifts, assuming an error on $d_{L}$ of $10 \%$. Lower plot: the combination of all events of the upper plot. We use as fiducial values $H_{0}=70 \mathrm{~km} \mathrm{~s}^{-1} \mathrm{Mpc}^{-1}$ and $\Xi_{0}=1.8$.

Planck 2018 values, would be at the sub-percent level, so, in practice, $\left(\Delta d_{L} / d_{L}\right)_{\Delta t}$ would be negligible and eq. (16) will be replaced by $\Delta d_{L} / d_{L} \simeq\left(\Delta d_{L} / d_{L}\right)_{\mathrm{GW}}$. Therefore, we will have a smaller value of $\Delta d_{L} / d_{L}$, at the price of having assumed a value for $H_{0}$.

On the opposite side, one might want to explore what limits can be set on both $H_{0}$ and $\Xi_{0}$ from a quadruply lensed event, without any prior on $H_{0}$. When opening up this two-dimensional parameter space, the degeneracy between $H_{0}$ and $\Xi_{0}$ from a $d_{L}^{\mathrm{gw}}$ measurement with known redshift $z_{s}$ can be broken without an external prior on $H_{0}$, since the $d_{L}^{\mathrm{em}}$ measurement constrains $H_{0}$. This is shown in the upper plot of of 5 , where we show five events at different redshifts, simulated with the same procedure performed for obtaining Figs. 1-3. The plot also shows that the $1 \sigma$ contours are ellipses whose tilt changes with $z_{s}$, so the combination of events at different redshifts helps to break the degeneracy further. The lower plot shows an example of the combined posteriors from the five events in the upper plot. From this example of simulated events, we get a combined result $\Xi_{0}=1.80_{-0.18}^{+0.16}$ and $H_{0}=70.3 \pm 3.1$, so our fiducial values are recovered to an accuracy of about $9 \%$ for $\Xi_{0}$ and $4 \%$ for $H_{0}$. Note that, to avoid cluttering the plot, we are again neglecting to simulate the noise that would scatter these ellipses according to their size. In practice, the result we present here for 5 events is achieved, and even improved upon, by half of a set of 10 real events alone, while the remaining half of events will only contribute insignificantly to the constraint.

\section{CONCLUSIONS}

The observation of strong lensing of GW events might already be possible for $2 \mathrm{G}$ detectors, and is expected to be common for $3 \mathrm{G}$ detectors. A fraction of these events (from $30 \%$ at $2 \mathrm{G}$ to $6 \%$ at $3 \mathrm{G}$ detectors) could be quadruply lensed. As discussed in [22], for these events it can be possible to localize the host galaxy even in the absence of an electromagnetic flare, and therefore these systems could be used as standard sirens. In this paper we have explored the consequences in the context of modified gravity, in particular for modified gravitational wave propagation. We have pointed out that, in such a context, a quadruply lensed system allows the simultaneous determination of the electromagnetic luminosity distance and of the gravitational-wave luminosity distance of the source. Quite interestingly, with this method one can obtain limits on $\Xi_{0}$, which is the most important parameter that enters in the parametrization (3) of modified GW propagation, without imposing a prior on $H_{0}$, and one can also perform a joint inference for $\left(H_{0}, \Xi_{0}\right)$.

Our main results are summarized in Fig. 3, that shows the relative error that can be obtained on $\Xi_{0}$, as a function of the redshift of the source and of the error on the measurements of the electromagnetic and gravitational luminosity distances (combined in quadrature). Comparing with the largest prediction available from a viable cosmological model, which is $\Xi_{0} \simeq 1.8$ for nonlocal gravity [39, 40], corresponding to a $80 \%$ deviation from the GR value $\Xi_{0}=1$, we see that significant results might already be obtained by $2 \mathrm{G}$ detectors, while a third-generation detector such as ET, combining the events observed over a few years, might reach a very interesting accuracy, possibly of order $6 \%$, on $\Xi_{0}$.

Acknowledgments. The work of the authors is supported by the Swiss National Science Foundation and by the SwissMap National Center for Competence in Research. 
[1] O. A. Hannuksela, K. Haris, K. K. Y. Ng, S. Kumar, A. K. Mehta, D. Keitel, T. G. F. Li, and P. Ajith, "Search for gravitational lensing signatures in LIGO-Virgo binary black hole events," Astrophys. J. Lett. 874 (2019) L2, arXiv:1901.02674 [gr-qc].

[2] A. K. Y. Li, R. K. L. Lo, S. Sachdev, C. L. Chan, E. T. Lin, T. G. F. Li, and A. J. Weinstein, "Finding diamonds in the rough: Targeted Sub-threshold Search for Strongly-lensed Gravitational-wave Events," arXiv: 1904.06020 [gr-qc].

[3] C. McIsaac, D. Keitel, T. Collett, I. Harry, S. Mozzon, O. Edy, and D. Bacon, "Search for strongly lensed counterpart images of binary black hole mergers in the first two LIGO observing runs," Phys. Rev. D 102 (2020) 084031, arXiv:1912.05389 [gr-qc].

[4] P. T. H. Pang, O. A. Hannuksela, T. Dietrich, G. Pagano, and I. W. Harry, "Lensed or not lensed: Determining lensing magnifications for binary neutron star mergers from a single detection," arXiv:2002.04893 [astro-ph.HE].

[5] X. Liu, I. M. Hernandez, and J. Creighton, "Identifying strong gravitational-wave lensing during the second observing run of Advanced LIGO and Advanced Virgo," Astrophys. J. 908 no. 1, (2021) 97, arXiv:2009.06539 [astro-ph.HE].

[6] L. Dai, B. Zackay, T. Venumadhav, J. Roulet, and M. Zaldarriaga, "Search for Lensed Gravitational Waves Including Morse Phase Information: An Intriguing Candidate in O2," arXiv:2007.12709 [astro-ph.HE].

[7] LIGO Scientific, Virgo Collaboration, R. Abbott et al., "Search for lensing signatures in the gravitational-wave observations from the first half of LIGO-Virgo's third observing run," arXiv:2105.06384 [gr-qc].

[8] J. M. Diego, T. Broadhurst, and G. Smoot, "Evidence for lensing of gravitational waves from LIGO-Virgo," arXiv:2106.06545 [gr-qc].

[9] K. K. Y. Ng, K. W. K. Wong, T. Broadhurst, and T. G. F. Li, "Precise LIGO Lensing Rate Predictions for Binary Black Holes," Phys. Rev. D 97 (2018) 023012, arXiv:1703.06319 [astro-ph.CO].

[10] S.-S. Li, S. Mao, Y. Zhao, and Y. Lu, "Gravitational lensing of gravitational waves: A statistical perspective," Mon. Not. Roy. Astron. Soc. 476 (2018) 2220-2229, arXiv: 1802.05089 [astro-ph.CO].

[11] M. Oguri, "Effect of gravitational lensing on the distribution of gravitational waves from distant binary black hole mergers," Mon. Not. Roy. Astron. Soc. 480 (2018) 3842-3855, arXiv:1807.02584 [astro-ph.C0].

[12] M. Punturo et al., "The Einstein Telescope: A third-generation gravitational wave observatory," Class. Quant. Grav. 27 (2010) 194002.

[13] M. Maggiore et al., "Science Case for the Einstein Telescope," JCAP 03 (2020) 050, arXiv:1912.02622 [astro-ph.CO].

[14] M. Biesiada, X. Ding, A. Piorkowska, and Z.-H. Zhu, "Strong gravitational lensing of gravitational waves from double compact binaries - perspectives for the Einstein Telescope," JCAP 10 (2014) 080, arXiv:1409.8360 [astro-ph.HE].

[15] X. Ding, M. Biesiada, and Z.-H. Zhu, "Strongly lensed gravitational waves from intrinsically faint double compact binaries - prediction for the Einstein Telescope," JCAP 12 (2015) 006, arXiv: 1508.05000 [astro-ph.HE].

[16] T. Treu, "Strong Lensing by Galaxies," Ann. Rev. Astron. Astrophys. 48 (2010) 87-125, arXiv:1003.5567 [astro-ph.CO].

[17] M. Bartelmann, "Gravitational Lensing," Class. Quant. Grav. 27 (2010) 233001, arXiv:1010.3829 [astro-ph. CO].

[18] M. Sereno, P. Jetzer, A. Sesana, and M. Volonteri, "Cosmography with strong lensing of LISA gravitational wave sources," Mon. Not. Roy. Astron. Soc. 415 (2011) 2773, arXiv:1104.1977 [astro-ph.CO].

[19] K. Liao, X.-L. Fan, X.-H. Ding, M. Biesiada, and Z.-H. $\mathrm{Zhu}$, "Precision cosmology from future lensed gravitational wave and electromagnetic signals," Nature Commun. 8 (2017) 1148, arXiv:1703.04151 [astro-ph.CO]. [Erratum: Nature Commun. 8, 2136 (2017)].

[20] B. Liu, Z. Li, and Z.-H. Zhu, "Complementary constraints on dark energy equation of state from strongly lensed gravitational wave," Mon. Not. Roy. Astron. Soc. 487 (2019) 1980-1985, arXiv:1904.11751 [astro-ph.CO].

[21] Y. Li, X. Fan, and L. Gou, "Constraining Cosmological Parameters in the FLRW Metric with Lensed GW+EM Signals," Astrophys. J. 873 no. 1, (2019) 37, arXiv:1901.10638 [astro-ph.CO].

[22] O. A. Hannuksela, T. E. Collett, M. Çalışkan, and T. G. F. Li, "Localizing merging black holes with sub-arcsecond precision using gravitational-wave lensing," Mon. Not. Roy. Astron. Soc. 498 (2020) 3395-3402, arXiv:2004.13811 [astro-ph.HE].

[23] I. D. Saltas, I. Sawicki, L. Amendola, and M. Kunz, "Anisotropic Stress as a Signature of Nonstandard Propagation of Gravitational Waves," Phys. Rev. Lett. 113 (2014) 191101, arXiv:1406.7139 [astro-ph.C0].

[24] L. Lombriser and A. Taylor, "Breaking a Dark Degeneracy with Gravitational Waves," JCAP 1603 (2016) 031, arXiv:1509.08458 [astro-ph.CO].

[25] A. Nishizawa, "Generalized framework for testing gravity with gravitational-wave propagation. I. Formulation," Phys. Rev. D97 (2018) 104037, arXiv: 1710.04825 [gr-qc].

[26] S. Arai and A. Nishizawa, "Generalized framework for testing gravity with gravitational-wave propagation. II. Constraints on Horndeski theory," Phys. Rev. D97 (2018) 104038, arXiv:1711.03776 [gr-qc].

[27] E. Belgacem, Y. Dirian, S. Foffa, and M. Maggiore, "The gravitational-wave luminosity distance in modified gravity theories," Phys. Rev. D97 (2018) 104066, arXiv:1712.08108 [astro-ph.CO].

[28] L. Amendola, I. Sawicki, M. Kunz, and I. D. Saltas, "Direct detection of gravitational waves can measure the time variation of the Planck mass," JCAP 1808 (2018) 030, arXiv:1712.08623 [astro-ph.CO].

[29] E. Belgacem, Y. Dirian, S. Foffa, and M. Maggiore, "Modified gravitational-wave propagation and standard sirens," Phys. Rev. D98 (2018) 023510, arXiv:1805.08731 [gr-qc]. 
[30] LISA Cosmology Working Group Collaboration, E. Belgacem et al., "Testing modified gravity at cosmological distances with LISA standard sirens," JCAP 1907 (2019) 024, arXiv:1906.01593 [astro-ph.CO].

[31] A. Finke, S. Foffa, F. Iacovelli, M. Maggiore, and M. Mancarella, "Cosmology with LIGO/Virgo dark sirens: Hubble parameter and modified gravitational wave propagation," arXiv:2101.12660 [astro-ph.CO].

[32] B. P. Abbott et al., "Gravitational Waves and Gamma-rays from a Binary Neutron Star Merger: GW170817 and GRB 170817A," Astrophys. J. 848 (2017) L13, arXiv:1710.05834 [astro-ph.HE].

[33] P. Creminelli and F. Vernizzi, "Dark Energy after GW170817 and GRB170817A," Phys. Rev. Lett. 119 (2017) 251302, arXiv:1710.05877 [astro-ph.CO].

[34] J. Sakstein and B. Jain, "Implications of the Neutron Star Merger GW170817 for Cosmological Scalar-Tensor Theories," Phys. Rev. Lett. 119 (2017) 251303, arXiv:1710.05893 [astro-ph.CO].

[35] J. M. Ezquiaga and M. Zumalacárregui, "Dark Energy After GW170817: Dead Ends and the Road Ahead," Phys. Rev. Lett. 119 (2017) 251304, arXiv:1710.05901 [astro-ph.CO].

[36] T. Baker, E. Bellini, P. G. Ferreira, M. Lagos, J. Noller, and I. Sawicki, "Strong constraints on cosmological gravity from GW170817 and GRB 170817A," Phys. Rev. Lett. 119 (2017) 251301, arXiv:1710.06394 [astro-ph.CO].

[37] M. Maggiore, "Phantom dark energy from nonlocal infrared modifications of general relativity," Phys.Rev. D89 (2014) 043008, arXiv: 1307.3898 [hep-th].

[38] M. Maggiore, "Nonlocal Infrared Modifications of Gravity. A Review," Fundam. Theor. Phys. 187 (2017) 221-281, arXiv:1606.08784 [hep-th].

[39] E. Belgacem, Y. Dirian, A. Finke, S. Foffa, and M. Maggiore, "Gravity in the infrared and effective nonlocal models," JCAP 04 (2020) 010, arXiv:2001.07619 [astro-ph.CO].

[40] E. Belgacem, Y. Dirian, A. Finke, S. Foffa, and M. Maggiore, "Nonlocal gravity and gravitational-wave observations," JCAP 1911 (2019) 022, arXiv: 1907.02047 [astro-ph.CO].

[41] M. Lagos, M. Fishbach, P. Landry, and D. E. Holz, "Standard sirens with a running Planck mass," Phys. Rev. D99 (2019) 083504, arXiv:1901.03321 [astro-ph.CO].

[42] G. Dálya, G. Galgóczi, L. Dobos, Z. Frei, I. S. Heng, R. Macas, C. Messenger, P. Raffai, and R. S. de Souza, "GLADE: A galaxy catalogue for multimessenger searches in the advanced gravitational-wave detector era," Mon. Not. Roy. Astron. Soc. 479 (2018) 2374-2381, arXiv:1804.05709 [astro-ph.HE].

[43] S. Mastrogiovanni, L. Haegel, C. Karathanasis, I. Magana-Hernandez, and D. Steer, "Gravitational wave friction in light of GW170817 and GW190521," arXiv:2010.04047 [gr-qc].

[44] S. Mastrogiovanni, D. Steer, and M. Barsuglia, "Probing modified gravity theories and cosmology using gravitational-waves and associated electromagnetic counterparts," Phys. Rev. D 102 (2020) 044009, arXiv:2004.01632 [gr-qc].

[45] J. M. Ezquiaga, "Hearing gravity from the cosmos: GWTC-2 probes general relativity at cosmological scales," arXiv:2104.05139 [astro-ph.CO].

[46] W. M. Farr, M. Fishbach, J. Ye, and D. Holz, "A Future Percent-Level Measurement of the Hubble Expansion at Redshift 0.8 With Advanced LIGO," Astrophys. J. Lett. 883 no. 2, (2019) L42, arXiv:1908.09084 [astro-ph.C0].

[47] D. Reitze et al., "Cosmic Explorer: The U.S. Contribution to Gravitational-Wave Astronomy beyond LIGO," Bull. Am. Astron. Soc. 51 (2019) 035, arXiv:1907.04833 [astro-ph.IM].

[48] P. Amaro-Seoane et al., "Laser Interferometer Space Antenna," arXiv:1702.00786 [astro-ph.IM].

[49] E. Belgacem, Y. Dirian, S. Foffa, E. J. Howell, M. Maggiore, and T. Regimbau, "Cosmology and dark energy from joint gravitational wave-GRB observations," JCAP 1908 (2019) 015, arXiv: 1907.01487 [astro-ph.CO].

[50] T. Baker and I. Harrison, "Constraining Scalar-Tensor Modified Gravity with Gravitational Waves and Large Scale Structure Surveys," JCAP 01 (2021) 068, arXiv:2007.13791 [astro-ph.CO].

[51] G. Pagano, O. A. Hannuksela, and T. G. F. Li, "lensingGW: a Python package for lensing of gravitational waves," Astron. Astrophys. 643 (2020) A167, arXiv:2006.12879 [astro-ph.CO].

[52] G. P. Smith, M. Jauzac, J. Veitch, W. M. Farr, R. Massey, and J. Richard, "What if LIGO's gravitational wave detections are strongly lensed by massive galaxy clusters?," Mon. Not. Roy. Astron. Soc. 475 (2018) 3823-3828, arXiv:1707.03412 [astro-ph.HE].

[53] K. Haris, A. K. Mehta, S. Kumar, T. Venumadhav, and P. Ajith, "Identifying strongly lensed gravitational wave signals from binary black hole mergers," arXiv:1807.07062 [gr-qc].

[54] P. Schneider, J. Ehlers, and E. E. Falco, Gravitational Lenses. Springer, 1992.

[55] S. Cao, J. Qi, M. Biesiada, T. Liu, J. Li, and Z.-H. Zhu, "Measuring the viscosity of dark matter with strongly lensed gravitational waves," Mon. Not. Roy. Astron. Soc. 502 (2021) L16-L20, arXiv:2012.12462 [astro-ph.CO].

[56] M. Chevallier and D. Polarski, "Accelerating universes with scaling dark matter," Int.J.Mod.Phys. D10 (2001) 213-224, arXiv:gr-qc/0009008 [gr-qc].

[57] E. V. Linder, "Exploring the expansion history of the universe," Phys.Rev.Lett. 90 (2003) 091301, arXiv:astro-ph/0208512 [astro-ph].

[58] C. Dalang and L. Lombriser, "Limitations on Standard Sirens tests of gravity from screening," JCAP 1910 (2019) 013, arXiv:1906.12333 [astro-ph.CO].

[59] Planck Collaboration, N. Aghanim et al., "Planck 2018 results. VI. Cosmological parameters," Astron. Astrophys. 641 (2020) A6, arXiv:1807.06209 [astro-ph.CO].

[60] DES Collaboration, T. M. C. Abbott et al., "Dark Energy Survey Year 1 Results: Constraints on Extended Cosmological Models from Galaxy Clustering and Weak Lensing," Phys. Rev. D99 (2019) 123505, arXiv:1810.02499 [astro-ph.CO].

[61] A. G. Riess, S. Casertano, W. Yuan, L. M. Macri, and D. Scolnic, "Large Magellanic Cloud Cepheid Standards Provide a $1 \%$ Foundation for the Determination of the Hubble Constant and Stronger Evidence for Physics 
Beyond LambdaCDM," Astrophys. J. 876 (2019) 85, arXiv:1903.07603 [astro-ph.CO].

[62] K. C. Wong et al., "H0LiCOW - XIII. A 2.4 per cent measurement of $\mathrm{H} 0$ from lensed quasars: $5.3 \sigma$ tension between early- and late-Universe probes," Mon. Not. Roy. Astron. Soc. 498 no. 1, (2020) 1420-1439, arXiv:1907.04869 [astro-ph.CO].

[63] A. G. Riess, S. Casertano, W. Yuan, J. B. Bowers, L. Macri, J. C. Zinn, and D. Scolnic, "Cosmic Distances Calibrated to 1\% Precision with Gaia EDR3 Parallaxes and Hubble Space Telescope Photometry of 75 Milky Way Cepheids Confirm Tension with $\Lambda$ CDM,"
Astrophys. J. Lett. 908 (2021) L6, arXiv:2012.08534 [astro-ph.CO].

[64] T. E. Collett, "The population of galaxy-galaxy strong lenses in forthcoming optical imaging surveys," Astrophys. J. 811 (2015) 20, arXiv:1507.02657 [astro-ph.CO].

[65] H.-Y. Chen, D. E. Holz, J. Miller, M. Evans, S. Vitale, and J. Creighton, "Distance measures in gravitational-wave astrophysics and cosmology," Class. Quant. Grav. 38 (2021) 055010, arXiv:1709.08079 [astro-ph. CO]. 